\title{
Análisis de Modelos de Lechos Fuente en Multietapa, utilizando la Distribución del Tiempo de Residencia de los Sólidos
}

\author{
Enrique Arriola-Guevara (1), Guadalupe M. Guatemala-Morales $(1,2)$, \\ Tania García-Herrera $(1,2)$ y Jorge A. García-Fajardo (2) \\ (1) Centro Universitario de Ciencias Exactas e Ingenierías, Universidad de Guadalajara, \\ Calzada Marcelino García Barragán y Olímpica, Sector Reforma, CP 44420 Guadalajara, \\ Jalisco-México (e-mail: arriole@hotmail.com) \\ (2) Centro de Investigación y Asistencia en Tecnología y Diseño del Estado de Jalisco, A.C., \\ Avenida Normalistas N 800, Colonia Colinas de la Normal, CP 44270 Guadalajara, Jalisco-México
}

\begin{abstract}
Resumen
Se presenta un análisis comparativo de modelos de flujo desarrollados para los sólidos de un sistema de lechos fuente en multietapa (multistage spouted bed) diseñado por Arriola (1997). Se seleccionaron modelos de distribución del tiempo de residencia de los sólidos adecuados para la aplicación comercial del sistema en el tratamiento de granos y semillas. La determinación experimental de la función de distribución del tiempo de residencia se llevó acabo mediante la técnica estímulorespuesta utilizando partículas coloreadas. Se compararon cuatro modelos: tres de compartimentos y el de tanques-en-serie. La comparación de esos modelos mostró que los de compartimentos son los más apropiados para aplicaciones comerciales. El análisis estadístico permite afirmar que el modelo más recomendable, debido a su simplicidad, es el modelo de compartimentos propuesto por Arriola.
\end{abstract}

Palabras clave: lechos fuente multietapa, tiempos de residencia, modelos de flujo, modelos de compartimiento

\section{Analysis of Models of Spouted Bed Multistage Systems using the Residence Time Distribution of the Solids}

\begin{abstract}
A comparative analysis of flow models developed for the solids in a multistage spouted bed system designed by Arriola (1997), is presented. Models for the residence time distribution, suitable for commercial applications in processing seeds and grains, has been selected in the study. The experimental residence time distribution function was obtained by the stimulus-response technique using colored particles. Four models were compared: three compartment models, and the stirred-tank-in-series model. Evaluation of these models showed that the compartment models are more suitable for commercial applications. The statistical analysis allows to conclude that the best model, due to its simplicity, is the compartment model proposed by Arriola.
\end{abstract}

Keywords: multistage spouted bed, residence time distribution, flow models, compartment models 


\section{INTRODUCCIÓN}

El lecho fuente es una técnica para el contacto de fluidos con partículas sólidas. Una de las mayores ventajas de estos sistemas de contacto fluido-sólido radica en el hecho de que son capaces de manejar partículas sólidas de gran tamaño $\left(>10^{-3} \mathrm{~m}\right)$, las que normalmente resultan muy difíciles de fluidizar. Los lechos fuente, que inicialmente se desarrollaron como un método para el secado de granos (Peterson, 1962; Clary et al, 1970), posteriormente se han utilizado en muchos otros procesos tales como: la separación de sólidos (Van Weert y Van Hasselt, 1997), la granulación (Tsvik, 1967), las reacciones catalíticas (Olazar et al., 1994), tostado de café (Nagaraju et al., 1997), tratamiento térmico para el control de insectos (Banks, 1998 y Beckett, 2000), recubrimiento de partículas (Jones, 1994), pirólisis de poliestireno (Aguado et al., 2003), secado de camarón (Tapaneyasin et al., 2005) y, en general, las operaciones de transferencia de calor y de masa (Uemaki y Kugo, 1967 y Epstein y Mathur, 1974, 1982). La gran mayoría de los diseños de lechos fuente hasta ahora reportados en operaciones por lote -por lo que a sólidos respecta- de una sola etapa, se refieren a lechos cilíndricos o semi-cilíndricos (Mann y Crosby, 1972; Nagaraju et al., 1997; Benali y Amazouz, 2002; Nagahashi et al., 2002; Bacelos et al., 2004; Souza y Oliveira, 2005; Tapaneyasin et al., 2005) o lechos fuente cónicos (Olazar et al., 1998; Wang et al., 2002; San José et al., 2005; Arandes et al., 2004; Chan et al., 2006). La operación de contacto fluido-sólido en un lecho fuente consiste en inyectar una corriente de fluido a alta velocidad que se mueve a través de un lecho de sólidos, empujándolos y forzándolos a subir por el centro del recipiente hasta alcanzar el nivel superior del lecho, en donde los sólidos después caen por efecto de la gravedad como una lluvia de partículas formando una fuente.

A pesar de que teóricamente se ha demostrado que los lechos fuente, con flujo continuo de sólidos, se comportan de una manera muy similar al modelo de "tanque agitado" (Epstein y Mathur, 1974), y de que un sistema de tanques agitados en serie se aproxima al flujo pistón cuando el número de tanques es grande (Levenspiel y Bischoff,1963), existen muy pocos trabajos publicados sobre el uso, operación y modelación de lechos fuente en multietapa (Elperin y Khokhlov,1965; Malek y
Walsh, 1966; Rovero y Watkison,1990; Van Weert y Van Hasselt, 1997).

Según Arriola (1997), "estos lechos fuente tienen una característica en común: son inestables hidrodinámicamente hablando y presentan serias desventajas en su diseño para su posible aplicación comercial". Este investigador diseñó un proceso novedoso de lechos fuente en multietapa (tres etapas) en contracorriente, que ha demostrado ser, dentro del rango de condiciones de operación experimentadas, un ingenioso sistema sin partes móviles, hidrodinámicamente estable, de baja caída de presión por etapa debido al uso de tubos de arrastre y con claras ventajas para su posible comercialización.

Para la descarga continua de las partículas sólidas se utilizó una válvula-L modificada cuyo funcionamiento -sobre el que no existe previa información- no ha sido aún propiamente documentado, requiriéndose una mayor investigación sobre este tipo de dispositivos. La Fig. 1 muestra un diagrama simplificado del sistema utilizado.

En este trabajo se utilizó la distribución del tiempo de residencia (RTD) de sólidos en los lechos como uno de los criterios de diseño, desarrollándose diversos modelos matemáticos para predecir el flujo de los mismos. La importancia de encontrar el modelo adecuado que represente mejor estos sistemas, radica en la futura aplicación a procesos muy específicos que se pretende dar a los mismos. Estos procesos involucran la transferencia de calor y masa, así como las reacciones químicas. El análisis aquí realizado viene a confirmar que el contacto gas-sólido en estos sistemas es muy eficiente y que el tratamiento de las partículas es más uniforme y continuo, lo que resulta muy conveniente para el procesamiento de granos como, por ejemplo, el secado de semillas y el tostado continuo de café.

\section{PROCEDIMIENTO EXPERIMENTAL}

Con el fin de obtener la mayor información posible del esquema de contacto de las partículas y el gas fuenteante dentro de cada lecho (el término "fuenteante" ha sido acuñado recientemente en la traducción al castellano de la Tercera Edición del libro "Ingeniería de las Reacciones Químicas de O. Levenspiel, 2004), se llevó a cabo un extenso programa 
de experimentos para la obtención de la distribución de tiempos de residencia (RTD), en lechos idénticos y en un esquema de montaje similar al realizado por Arriola (1997) en una y tres etapas. Como fluido se utilizó aire a temperatura ambiente, como sólidos se utilizaron esferas de vidrio transparentes $(1.78 \mathrm{~mm}$ de diámetro y densidad de $2.64 \mathrm{~g} / \mathrm{cm}^{3}$ ) y como rastreador esferas de vidrio coloreadas. Los flujos de operación del aire y sólidos utilizados oscilaron entre 0 y $3.7 \mathrm{l} / \mathrm{s}$, y 0 a $17 \mathrm{~g} / \mathrm{s}$ respec- tivamente. Se realizaron múltiples experimentos de los llamados estímulo-respuesta, obteniéndose datos de concentración (C), masa de rastreador $(\mathrm{M})$, peso total del lecho $(\mathrm{W})$, velocidad de flujo volumétrico del gas (FVG) y velocidad de flujo másico de sólidos $\left(\mathrm{S}_{\mathrm{o}}\right)$. A partir de esta información se construyeron gráficas de distribución de tiempos de residencia, $\mathrm{E}(\mathrm{t})$ y se obtuvieron valores del tiempo promedio de residencia $(\tau)$.

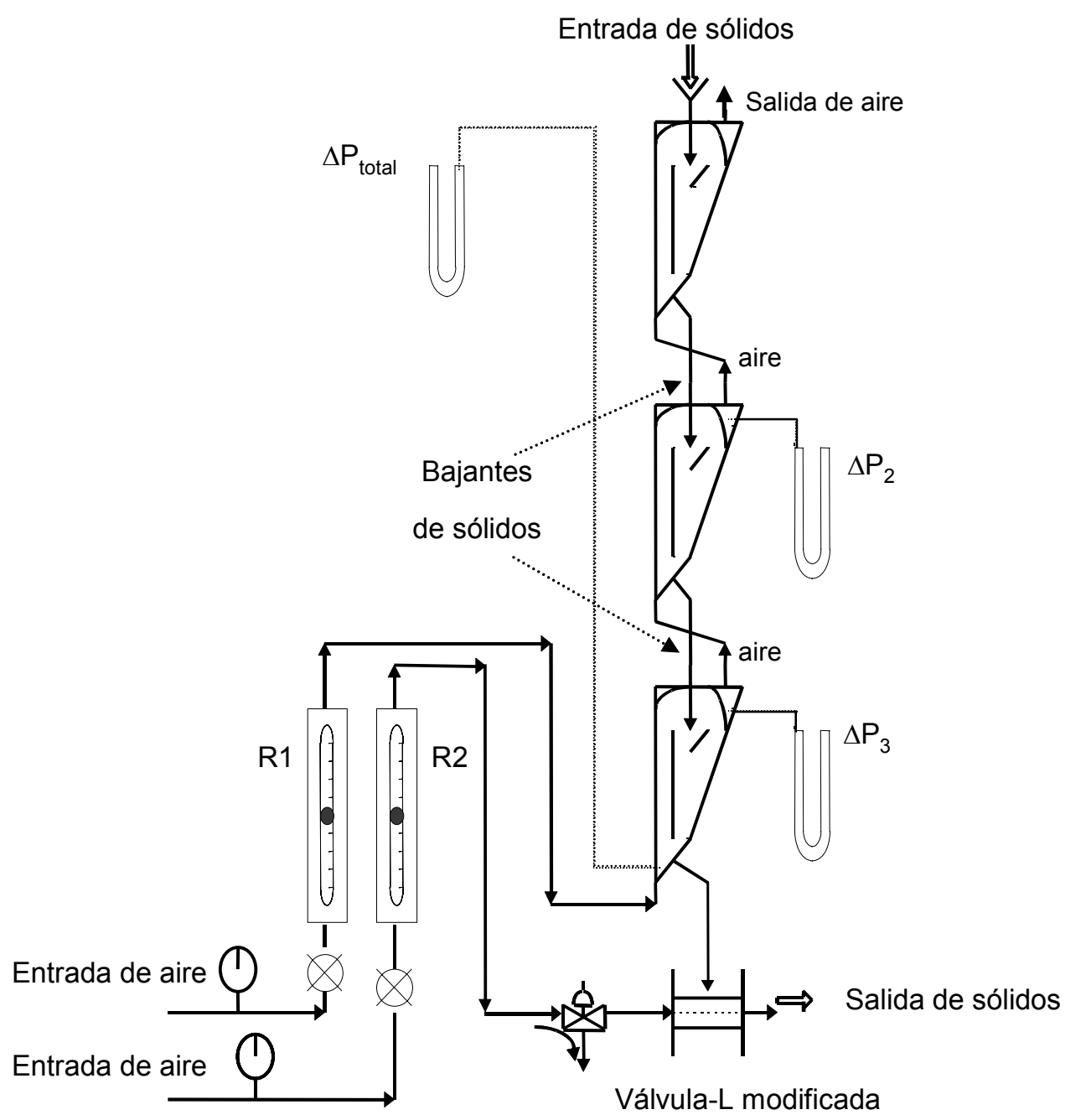

Fig. 1: Diagrama de una columna de tres etapas. Sistema diseñado por Arriola, 1997.

El sistema funciona de la siguiente manera: los sólidos son alimentados y extraídos por tubos de igual diámetro; las partículas sólidas entran por gravedad al recipiente, llenándolo hasta la altura del tubo de arrastre gracias a un deflector que, ingeniosamente colocado, dirige a los sólidos hacia la parte derecha del tubo de arrastre. Dependiendo del flujo de salida, controlado en el fondo por la válvula- $L$ modificada, será el tiempo que estos permanezcan circulando dentro de los recipientes. El aire, o fluido fuenteante, entra por el vértice inferior de los recipientes, empujando a los sólidos y forzándolos a saltar por encima del tubo de arrastre, formando la fuente característica. Una malla fina, colocada estratégicamente, impide el paso de sólidos -o polvos finos producto de la trituración o desgaste- a espacios que afectarían el buen funcionamiento del sistema.

Para la determinación experimental de la función RTD se siguieron las etapas siguientes: 
1. Al tiempo $t=0$, se introdujeron $50 \mathrm{~g}$ de rastreador. Las partículas de rastreador entraron al recipiente junto con los sólidos que ingresaron al sistema. Al mismo tiempo, se empezaron a tomar muestras a la salida del sistema.

2. Las muestras se tomaron a intervalos regulares de tiempo, por ejemplo, cada 5,10, 15 segundos.

3. Cuando se consideró que todas las partículas de rastreador salieron del sistema, se detuvo la entrada y salida de los flujos de sólidos y se cerró la válvula del aire fuenteante.

4. Se permitió la salida de los sólidos que quedaron en el sistema y se comprobó el balance de materia del rastreador.

Después de recolectar todas las muestras producidas de cada experimento, se procedió al manejo de cada una de la forma indicada a continuación:

5. Se pesaron las muestras y el contenido del lecho(s).

6. En cada muestra, se separó el rastreador del resto de las partículas.

\section{Se pesó el rastreador de cada muestra.}

8. Se separaron y se pesaron las partículas de rastreador que permanecieron dentro del lecho después que finalizó el experimento.

El lecho fuente, o sistema de lechos fuente, permite la observación del movimiento de las partículas dentro del(los) recipiente(s) ya que está(n) construido(s) de paredes de acrílico transparente. Es posible entonces visualizar e identificar, claramente, ciertas zonas en el movimiento de los sólidos dentro del sistema, por ejemplo: zonas con un movimiento descendente uniforme que indican la presencia del flujo pistón, zonas con turbulencia (en mayor o menor grado), que corresponderían a tanques agitados y, como consecuencia de la geometría muy característica de este sistema, zonas que recuerdan el flujo laminar.

\section{DESARROLLO DE MODELOS}

Se han desarrollado diferentes modelos para, por ejemplo, predecir la trayectoria del flujo de fluidos, la velocidad mínima de fuenteado, la caída de presión en columnas de acrílico ci- líndricas y semicilíndricas en sistemas de lechos fuente de una etapa (Grbavcic et al., 1976); modelos de RTD para el gas (Lim y Mathur, 1976; Olazar et al.,1993); modelos para describir el flujo asimétrico en el ánulo (Littman et al., 1985) o el comportamiento dinámico del fluido y la partícula (Krzywanski et al., 1992) y, finalmente, modelos empíricos de varias etapas para calcular la velocidad mínima de fuenteado (Murthy y Singh, 1994). Todos los sistemas que se mencionan, son geométrica e hidrodinámicamente diferentes al aquí analizado; asimismo, los modelos RTD presentados en este trabajo se generan de la observación de las regiones de flujo de los sólidos en los recipientes.

\section{Modelos de compartimentos}

El primer modelo fue el propuesto por Arriola (1997); este modelo está integrado por un recipiente de flujo pistón seguido de un tanque agitado (CSTV). La Fig. 2 muestra las zonas identificadas.

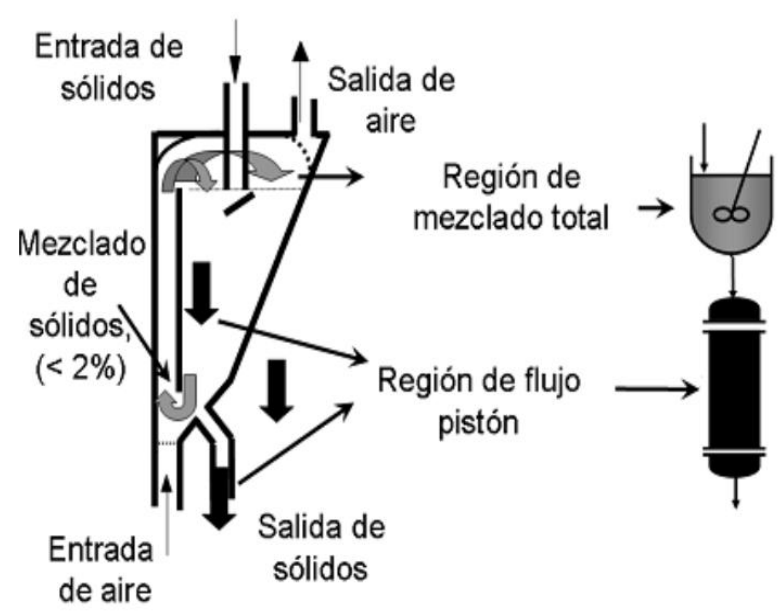

Fig. 2: Esquema del primer modelo de compartimentos: zonas de flujo observadas e identificadas por Arriola (1997).

Para obtener la función $E(t)$ a la salida del modelo propuesto, se hace uso de la convolución matemática, obteniendo así las ecuaciones (1) a la (7). La función RTD a la salida del modelo propuesto, $E(t)_{1}$, para una etapa es:

$\mathrm{E}(\mathrm{t})_{1}=\mathrm{E}(\mathrm{t})_{\mathrm{p}} * \mathrm{E}(\mathrm{t})_{\mathrm{M}}$

Donde $\tau_{\mathrm{p}}$ y $\tau_{\mathrm{M}}$, representan el tiempo de residencia de los recipientes de flujo pistón y tanque agitado respectivamente. 
Como se tiene que:

$\mathrm{E}(\mathrm{t})_{1}=0 \quad$ para $\mathrm{t}<\tau_{\mathrm{p}}$

$E(t)_{1}=\frac{1}{\tau_{M}} \exp \left[\frac{-\left(t-\tau_{p}\right)}{\tau_{M}}\right] \quad$ para $t \geq \tau_{p}$

Con la consideración de etapas idénticas, al convolucionar consigo misma la expresión anterior, se obtiene:

$\mathrm{E}(\mathrm{t})_{3}=0$

para $\mathrm{t}<3 \tau_{\mathrm{p}}$

$E(t)_{3}=\frac{1}{2 \tau_{M}^{3}}\left(t-3 \tau_{p}\right)^{2} \exp \left[\frac{-\left(t-3 \tau_{p}\right)}{\tau_{M}}\right]$

Para $\mathrm{t} \geq 3 \tau_{\mathrm{p}}$

y para "n" etapas:

$\mathrm{E}(\mathrm{t})_{\mathrm{n}}=0 \quad$ para $\mathrm{t}<\mathrm{n} \tau_{\mathrm{p}}$

$E(t)_{n}=\frac{1}{(n-1) \tau_{M}^{n}}\left(t-3 \tau_{p}\right)^{n-1} \exp \left[-\frac{\left(t-n \tau_{p}\right)}{\tau_{M}}\right]$

Para $\mathrm{t} \geq \mathrm{n} \tau_{\mathrm{p}}$

El segundo modelo de compartimentos, propuesto por Guatemala (2001), es un modelo un poco más complejo: incluye un recipiente de flujo laminar, por la deformación al flujo causada por la geometría del lecho, un tanque agitado continuo y un recipiente de flujo pistón, tal como se muestra en la Fig. 3.

Para una etapa, la convolución respectiva de las funciones RTD, para los elementos de flujo anteriormente mencionados está dada por la ecn. (8):

$E(t)_{1}=E(t)_{\operatorname{lam}}^{*} E(t)_{M}^{*} E(t)_{p}$

y debe cumplirse que,

$$
\begin{array}{ll}
E(t)_{\text {lam }}=0 & t<\frac{\tau_{\text {lam }}}{2} \\
E(t)_{\text {lam }}=\frac{\tau_{\text {lam }}^{2}}{t^{3}} & t \geq \frac{\tau_{\text {lam }}}{2} \\
E(t)_{M}=\frac{e^{-t / \tau_{M}}}{\tau_{M}} & t \geq \frac{\tau_{\text {lam }}}{2}
\end{array}
$$

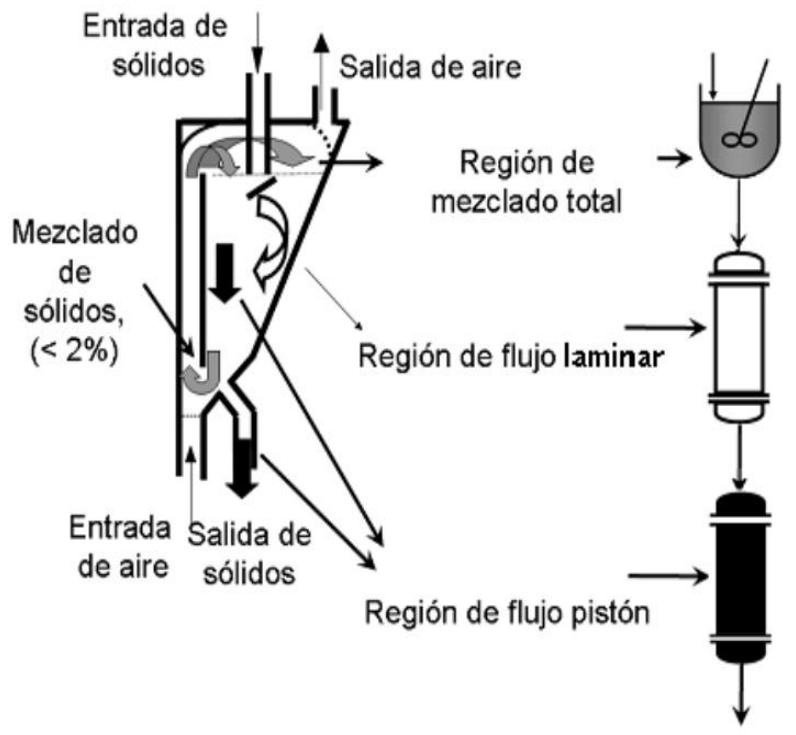

Fig. 3: Zonas observadas e identificadas por Guatemala (2001); esquema del modelo \#2.

$$
\begin{array}{ll}
E(t)=\delta(t) & t=0 \\
E(t)_{p}=\delta\left(t-\tau_{p}\right) & t=\tau_{p}
\end{array}
$$

Obteniéndose:

$$
\begin{aligned}
& E(t)_{1}=\frac{\tau_{\text {lam }}^{2}}{4 \tau_{M}}\left[\exp ^{-(t-c) / \tau_{M}}\right]\left[A-\left(\frac{1}{\tau_{M}}\right)^{2} B\right] \\
& \text { para } \\
& \mathrm{t} \geq \mathrm{C}=\left(\tau_{\mathrm{p}}+\frac{\tau_{\mathrm{lam}}}{2}\right) \\
& \text { y } \quad \mathrm{E}(\mathrm{t})_{1}=0 \text { para } \mathrm{t}<\mathrm{C}=\left(\tau_{\mathrm{p}}+\frac{\tau_{\mathrm{lam}}}{2}\right)
\end{aligned}
$$

Las constantes A y B están dadas por:

$$
A=\left(\frac{\exp \left(\frac{\tau_{\text {lam }}}{2} \tau_{M}\right)}{\left(\frac{\tau_{\text {lam }}}{2}\right)^{2}}+\frac{\exp \left(\frac{\tau_{\text {lam }}}{2} \tau_{M}\right.}{\tau_{M}\left(\frac{\tau_{\text {lam }}}{2}\right)}\right)
$$

$\left.\mathrm{B}=\left(\ln \left(\frac{\tau_{\mathrm{lam}}}{2}\right)+\frac{\left(\frac{\tau_{\mathrm{lam}}}{2}\right)}{\tau_{\mathrm{M}}}+\frac{\left(\frac{\tau_{\mathrm{lam}}}{2} \tau_{\mathrm{M}}\right.}{2.2 !}+\frac{\left(\left(\frac{\tau_{\mathrm{lam}}}{2}\right)\right.}{\tau_{\mathrm{M}}}\right)^{3}\right)$ 
Asimismo, si se convoluciona la ecn. (14) dos veces consigo misma, se obtiene la ecuación (19), correspondiente a la columna de tres etapas:

$E(t)_{3}=\frac{(t-3 C)^{2}\left(\exp ^{-\left(t-C / \tau_{M}\right.}\right)\left(\frac{\tau_{\text {lam }}^{2}}{4 \tau_{M}}\left(A-\left(\frac{1}{\tau_{M}}\right)^{2} B\right)\right)^{3}}{2 !}$

Para $\quad \mathrm{t} \geq 3 \mathrm{C}=3\left(\tau_{\mathrm{p}}+\frac{\tau_{\mathrm{lam}}}{2}\right)$

$y E(t)_{3}=0$ para $t<3 C=3\left(\tau_{p}+\frac{\tau_{\text {lam }}}{2}\right)$

Realizando la convolución de la ecn. (14) para "n etapas", se obtiene la solución analítica que se muestra en la ecn. (22).

El tercer modelo de compartimentos, propuesto por Arriola et al., (2004), propone dos tanques agitados en paralelo con diferentes tiempos de residencia, que toma en cuenta dos regiones de mezclado diferentes, como lo muestra la Fig. 4.

$E(t)=\frac{\left.(t-n C)^{n-1}\right)\left(\exp ^{-\left(t-C \tau_{M}\right)}\right)\left(\frac{\tau_{\text {lam }}^{2}}{4 \tau_{M}}\left(A-\left(\frac{1}{\tau_{M}}\right)^{2} B\right)\right)^{n}}{(n-1) !}$

Para $\mathrm{t} \geq \mathrm{nC}$

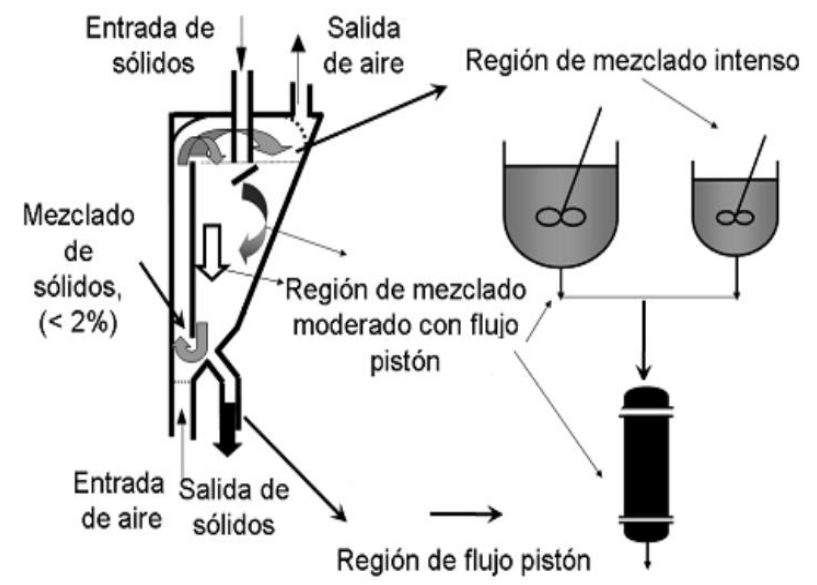

Fig. 4: Zonas observadas e identificadas por Arriola et al. (2004); esquema del modelo \#3.

La función de distribución de tiempos de resi- dencia para una etapa, con este esquema, se muestra en la ecn. (23).

$E(t)_{1}=\left[\frac{x}{\tau_{M 1}} \exp \left(-\frac{t-\tau_{P}}{\tau_{M 1}}\right)+\frac{1-x}{\tau_{M 2}} \exp \left(-\frac{t-\tau_{P}}{\tau_{M 2}}\right)\right]$

para $t>\tau_{p}$

Donde $\mathrm{x}$ representa la fracción del flujo que se dirige al primer CSTV. El tiempo promedio de residencia esta dado como: $\tau=\tau_{M}+\tau_{P}$. Se puede demostrar que de un balance de material se obtiene:

$$
\tau_{M}=x \tau_{M 1}+(1-x) \tau_{M 2}
$$

La fracción de volumen del primer CSTV se calcula de:

$r=\frac{x \tau_{M 1}}{\tau_{R}}=\frac{\tau_{M 1}^{0}}{\tau_{R}^{0}}$

Donde, $\tau_{\mathrm{M} 1}^{0}$, es el tiempo de residencia en el tanque agitado a las condiciones de un flujo volumétrico del gas con valor nulo ( $F V G=0)$. El tiempo de residencia total, real, en el lecho fuente, con ese valor de FVG es, $\tau_{R}^{0}$. La fracción de flujo, $x$, a través de cada tanque es tomada como un parámetro del modelo. Sin embargo, la fracción volumen del primer tanque agitado es calculada para un $F V G=0$ para una y tres etapas y tomando como un valor fijo ( $r$ ) para cada sistema. Por esta razón, $x$, no es un parámetro independiente, ya que es determinado por la ecn. (25); así, los únicos parámetros de modelo son: $\tau_{\mathrm{M} 1}, \tau_{\mathrm{M} 2} \mathrm{y}$ $\tau_{\mathrm{p}}$. Para obtener la convolución consigo misma de la ecn. (23), necesaria para analizar los datos experimentales provenientes de la columna de tres etapas, se hizo uso de las Transformadas de Laplace, lo que permitió realizar la convolución de los CSTV en paralelo de las tres etapas primero, y posteriormente convolucionar el resultado con los tres recipientes de flujo pistón. La solución analítica es presentada en Arriola et al., (2004) como en la ecn. (26).

Ambas funciones RTD, para una y tres etapas, son utilizadas para representar los datos experimentales. Es importante aclarar que a, $b, c, d, e, y$ f no son parámetros ajustables del 
modelo, sino coeficientes constantes obtenidos de la representación de la fracción parcial. Los parámetros del sistema $\tau_{\mathrm{M} 1}, \tau_{\mathrm{M} 2}, \tau_{\mathrm{p}} \mathrm{y}$ $\mathrm{x}$; están sujetos a la restricción de la primera fracción, $r$, de volumen del CSTV. Esto lo convierte en un sistema de tres parámetros, cuyo valor se escoge al minimizar la suma de los residuos al cuadrado en cada grupo de datos.

$E(t)_{3}=\left[\begin{array}{l}\left(\frac{a}{\tau_{M_{1}}}+\frac{b\left(t-3 \tau_{p}\right)}{\tau_{M_{1}}{ }^{2}}+\frac{c\left(t-3 \tau_{p}\right)^{2}}{2 \tau_{M_{1}}{ }^{3}}\right) \exp \left(-\frac{\left(t-3 \tau_{p}\right)}{\tau_{M_{1}}}\right)+ \\ \left(\frac{d}{\tau_{M_{2}}}+\frac{e\left(t-3 \tau_{p}\right)}{\tau_{M_{2}}{ }^{2}}+\frac{f\left(t-3 \tau_{p}\right)^{2}}{2 \tau_{M_{2}}{ }^{3}}\right) \exp \left(-\frac{\left(t-3 \tau_{p}\right)}{\tau_{M_{2}}}\right)\end{array}\right]$

Para $t>3 \tau_{p}$

\section{Modelo de tanques-en-serie}

En este modelo, el número de tanques, $\mathrm{N}$, es el parámetro ajustable, su valor describe el grado de mezclado en el recipiente. A medida que $\mathrm{N}$ se incrementa, desde 1 hasta $\infty$, el flujo en el recipiente varía desde el tanque agitado hasta el flujo pistón. Las ecuaciones (27), derivadas inicialmente por McMullin y Weber (Levenspiel, 1993), son un resumen de las funciones RTD, las medias y las varianzas, tanto en tiempo normal como en tiempo adimensional.

$$
\begin{aligned}
& \overline{\mathrm{t}} \mathrm{E}=\left(\frac{\mathrm{t}}{\overline{\mathrm{t}}}\right)^{\mathrm{N}-1} \frac{\mathrm{N}^{N}}{(\mathrm{~N}-1) !} \exp (-\mathrm{t} N / \overline{\mathrm{t}}) \quad \cdots \overline{\mathrm{t}}=\mathrm{N} \overline{\mathrm{t}}_{\mathrm{i}} \cdots \sigma^{2}=\frac{\overline{\mathrm{t}}^{2}}{\mathrm{~N}} \\
& \bar{t}_{i} E=\left(\frac{t}{\overline{t_{i}}}\right)^{N-1} \frac{1}{(N-1) !} \exp \left(-t / \bar{t}_{i}\right) \quad \cdots \bar{t}_{i}=\frac{\bar{t}}{N} \cdots \sigma^{2}=N \bar{t}_{i}^{2} \\
& E_{\theta i}=\bar{t}_{i} N=\frac{\theta_{i}^{N-1}}{(N-1) !} \exp \left(-\theta_{i}\right) \quad \cdots \sigma_{\theta i}^{2}=N \\
& E_{\theta}=\left(\bar{t}_{i} N\right) E=N \frac{(N \theta)^{N-1}}{(N-1) !} \exp (-N \theta) \cdots \sigma_{\theta}^{2}=\frac{1}{N}
\end{aligned}
$$

\section{Modelo de Dispersión}

El modelo de dispersión, al igual que el de tanques en serie, proporciona información sobre el grado de mezclado que tienen las partículas dentro del recipiente en estudio. De acuerdo con Levenspiel (2004), ambos modelos "son más o menos equivalentes" por lo que sugiere al interesado "utilizar el que le resulte más práctico".

El parámetro fundamental de este modelo es el módulo de dispersión D/uL, que se determina de manera análoga al parámetro del modelo de tanques en serie, por medio de datos de la función $\mathrm{E}(\mathrm{t})$. Las características de flujo en este modelo pueden cambiar desde el extremo de flujo pistón ideal, (D/uL) $\rightarrow 0$, hasta el de flujo en tanque agitado, (D/uL) $\rightarrow \infty$. $\mathrm{Si}$ el flujo se desvía enormemente del flujo pistón, es muy probable que el recipiente real no cumpla las hipótesis del modelo y que existan una gran cantidad de fluctuaciones al azar independientes. En este caso es incluso cuestionable si debería utilizarse el modelo (Levenspiel, 2004).

En virtud de las características de flujo observadas en el sistema que modelamos (fluctuaciones, curvas de concentración del rastreador asimétricas o prolongadas), no se consideró práctico el uso de este modelo.

\section{DISCUSIÓN DE LOS RESULTADOS}

\section{Modelos de compartimentos}

Para predecir los parámetros de los diferentes modelos, de una y tres etapas, se utilizó el método de Gauss-Newton que es un algoritmo para minimizar la suma de los cuadrados de los residuos entre los datos experimentales y aquellos provenientes de las ecuaciones no-lineales. La precisión obtenida con este método es de 0.000001 , mientras que la tolerancia es del $2 \%$.

En la Fig. 5 se muestran las curvas teóricas de los tres modelos de compartimentos, que se obtienen por optimización de los parámetros para una etapa.

De manera similar se obtuvieron las curvas teóricas de los tres modelos de compartimentos, que se obtienen por optimización de los parámetros para tres etapas, los que se pueden apreciar en la Fig. 6. Para los experimentos con una columna de una etapa en condiciones de bajos flujos de gas $(3.2 \mathrm{l} / \mathrm{s})$ y de sólidos (3 $\mathrm{g} / \mathrm{s})$, se puede observar que las curvas de los tres modelos representan de 
manera aceptable la mayoría de los datos experimentales (Fig. 5). Sin embargo, para la columna de tres etapas (Fig. 6), con bajos flujos de gas $(3 \mathrm{l} / \mathrm{s})$ y altos flujos de sólidos (17 g/s), es posible apreciar un mejor ajuste de los datos con los modelos de Guatemala (2001) y Arriola et al., (2004).

Según Guatemala (2001), cuando aumenta el número de etapas, el porcentaje del volumen del recipiente de flujo laminar tiende a desaparecer y en algunos casos se hace cero. Sin embargo, cuando se trata de una sola etapa, el modelo es más realista, ya que muestra la influencia de la geometría del recipiente que es una característica única y distintiva del sistema. Según la prueba de rangos múltiples, no hay diferencia-estadísticamente significativaentre cualquier par de medias, para una confianza del 95\%. La desventaja del modelo de Arriola et al. (2004), sobre el de Guatemala (2001) y el de Arriola (1997), es que los dos últimos modelos pueden emplearse para predecir el comportamiento de un sistema de " $n$ etapas" bajo cualquier condición de operación, mientras que el primero, sólo es útil para modelar el sistema hasta tres etapas; intentar aplicarlo a las "n etapas" resultaría un trabajo sumamente complejo.

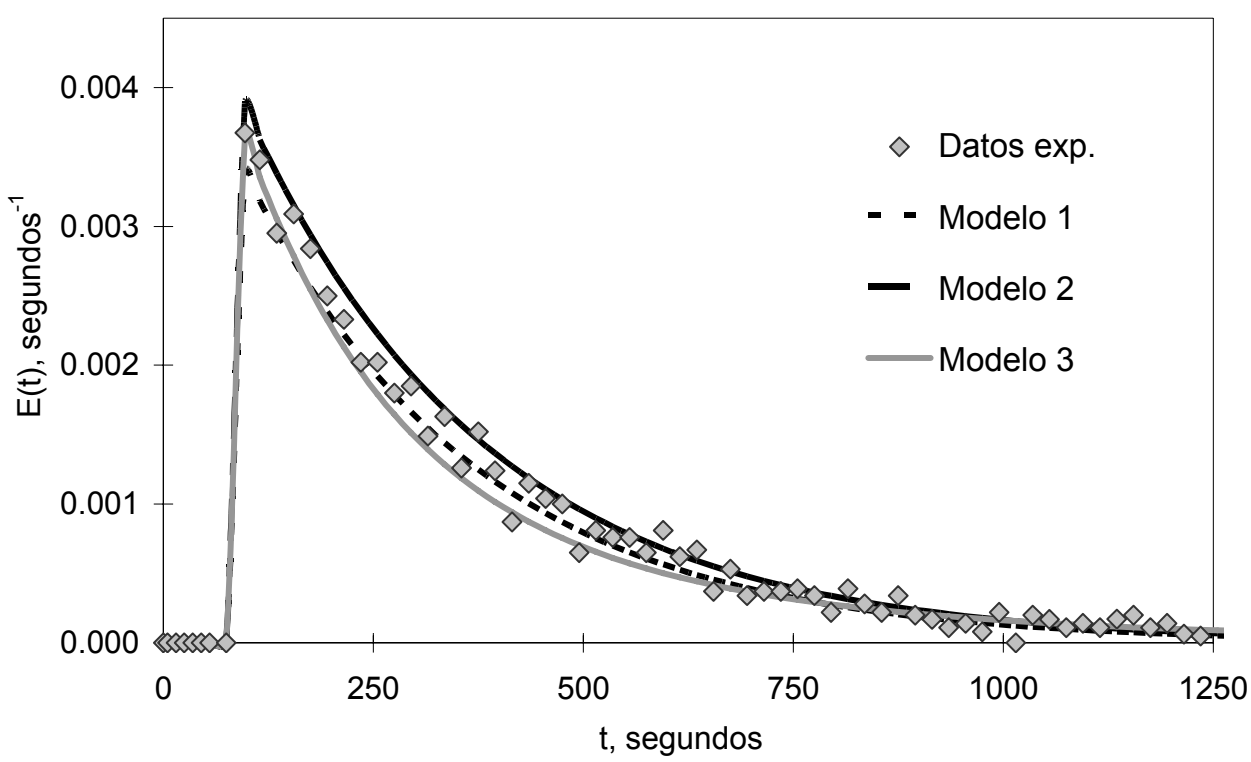

Fig. 5: Función RTD y modelos de compartimentos para una etapa. Modelo1 (Arriola, 1997); Modelo 2 (Guatemala, 2001); Modelo 3 (Arriola et al., 2004).

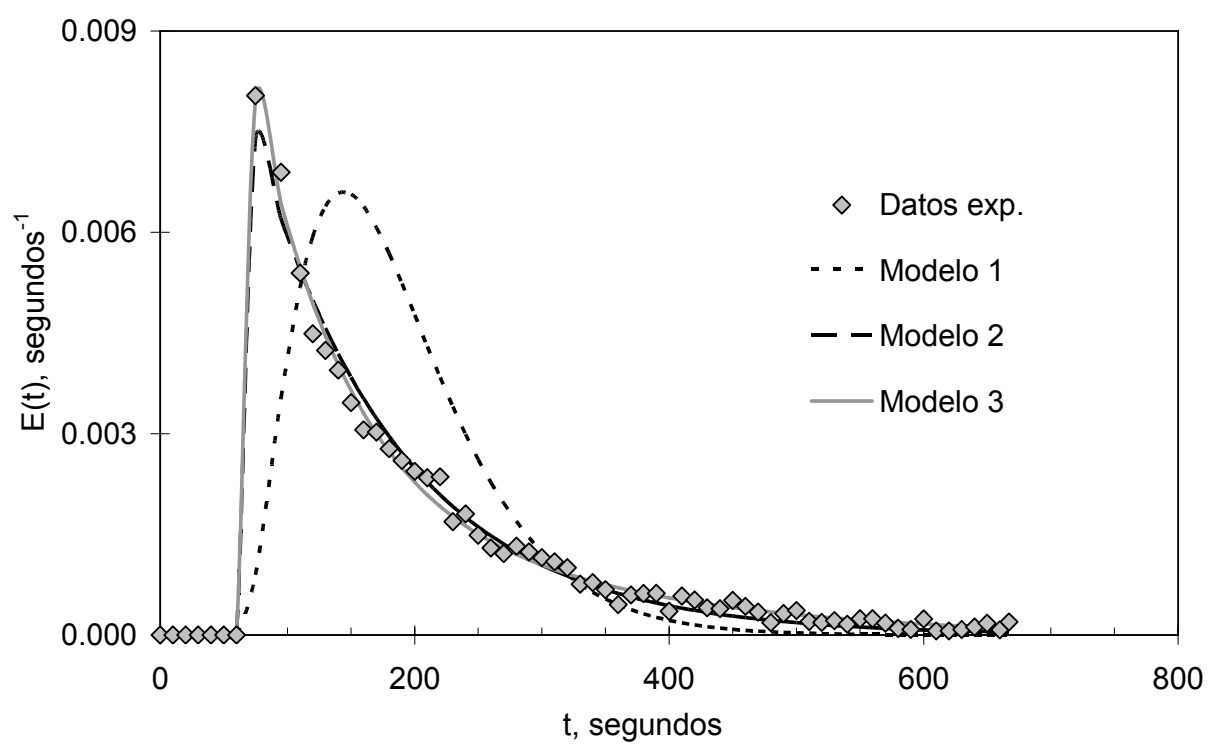

Fig. 6: Función RTD y modelos de compartimentos para tres etapas. Modelo1 (Arriola, 1997); Modelo 2 (Guatemala, 2001); Modelo 3 (Arriola et al., 2004). 


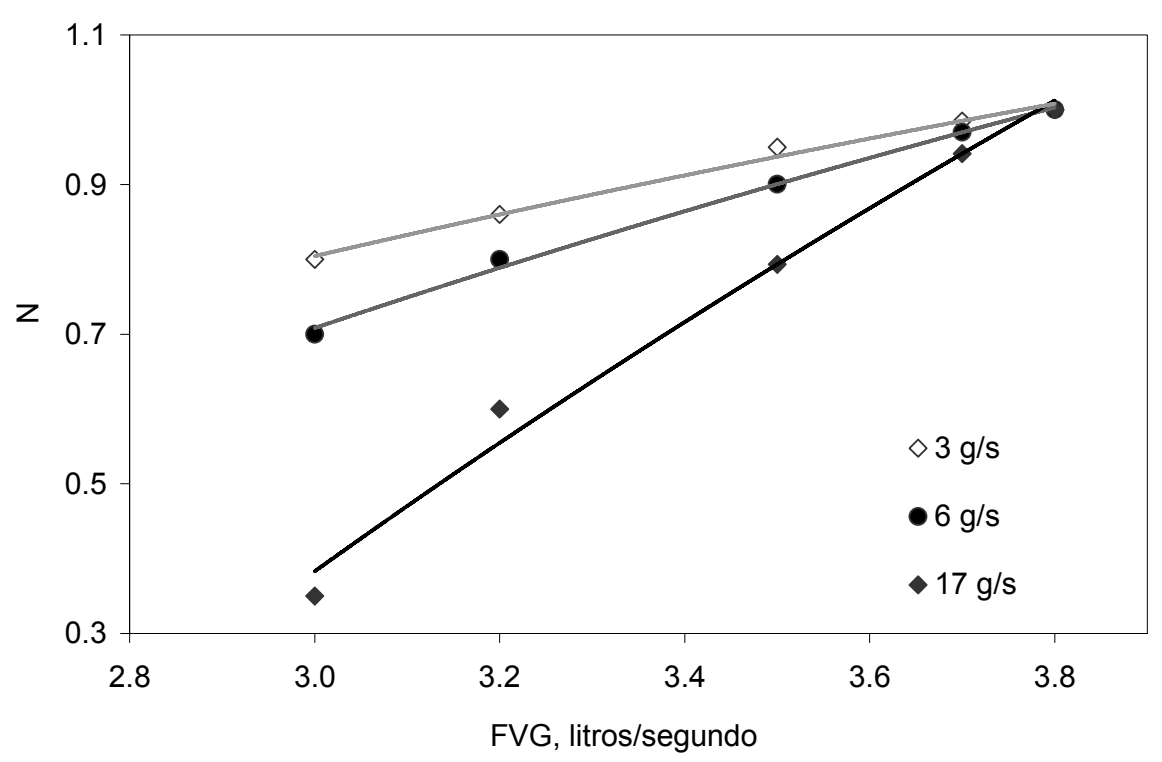

Fig. 7: Número de tanques en serie, $\mathrm{N}$, como función de los flujos de gas y sólidos. $\diamond 3 \mathrm{~g} / \mathrm{s}, \mathrm{y}=0.862 \ln (\mathrm{x})-0.1426, \mathrm{R}^{2}=0.9916 ; \bullet 6 \mathrm{~g} / \mathrm{s}, \mathrm{y}=1.2474$ $\ln (x)-0.6622, R^{2}=0.9967 ;-17 \mathrm{~g} / \mathrm{s}, \mathrm{y}=2.6628 \ln (\mathrm{x})-2.5425, \mathrm{R}^{2}=0.9884$.

Los modelos de compartimentos, resultan de gran utilidad como modelos modulares: una vez que se obtiene la expresión matemática para una etapa, mediante la convolución es posible generar una expresión para predecir el comportamiento de un sistema de "n etapas" para cualquier condición de operación. Por esta razón, es claro que de los modelos aquí analizados, los modelos de compartimentos son los más recomendables para su aplicación a columnas comerciales.

\section{Modelo de tanques en serie}

En cada experimento se determinó el tiempo promedio de residencia del rastreador y la desviación estándar del mismo; con estos valores se puede calcular mediante las ecns. (27) el valor de "N". En la Fig. 7, se muestran los valores de $\mathrm{N}$ en función de la velocidad del flujo volumétrico del gas, para diferentes velocidades de flujo másico de sólidos $S_{0}$. A baja velocidad del gas y alto flujo de sólidos, el mezclado es muy pobre; si se aumenta el flujo de gas y se disminuye el de sólidos, el efecto es el contrario, lo que es físicamente observable. Para valores elevados del flujo de gas, se produce una agitación intensa y el lecho fuente tiende a comportarse como un tanque agitado teórico.

Con la información de la Fig. 7 es posible obtener una correlación general que prediga la agitación, $\mathrm{N}$, en función de los flujos de gas y sólidos. Haciendo uso del método de Davis (1962) mostrado en Arriola (1997), se obtiene la ecn. (28).

$$
\begin{aligned}
N= & \left(0.1287 S_{0}+0.4756\right) * \ln (F V G)- \\
& \left(0.1713 S_{0}-0.3688\right)
\end{aligned}
$$

Con la ecn. (28) se obtiene la familia de curvas mostrada en la Fig. 8. De la misma manera, para una columna de tres etapas, la Fig. 9 representa el número de tanques en serie en función de los flujos de gas y sólidos. Siguiendo el mismo procedimiento indicado anteriormente para una etapa, se obtiene la correspondiente expresión para la columna de tres etapas:

$$
\begin{aligned}
N= & \left(0.0592 S_{0}+8.1024\right) * \ln (F V G)- \\
& \left(0.1439 S_{0}+6.4293\right)
\end{aligned}
$$

Como en el caso anterior, la ecn. (29) permite obtener la familia de curvas para diferentes valores supuestos de $S_{0}$, como se muestra en la Fig. 10. Pudiera pensarse que una vez que se obtiene una correlación como la ecuación anterior, se estaría en capacidad de predecir el comportamiento de columnas con otro número de etapas; desafortunadamente, el modelo de tanques-en-serie no permite este tipo de predicciones, que serían de gran utilidad, en virtud de que este no es un modelo modular. Esto quiere decir que cada vez que se pretenda aplicar el modelo a cada caso en particular, se tendrán que hacer los corres- 
pondientes experimentos de distribución del tiempo de residencia y analizar estos según lo que aquí se ha indicado. La aplicación de este modelo es limitada entonces a las condiciones en las que se obtienen los datos experimentales, por lo que su uso puede decirse que es sólo de carácter académico. Aunque el modelo de tanques-en-serie puede dar una idea rápida del grado de mezclado de los sólidos dentro del recipiente, este modelo no es adecuado para predecir las características de salida, cuando se cambia el número de etapas del sistema.

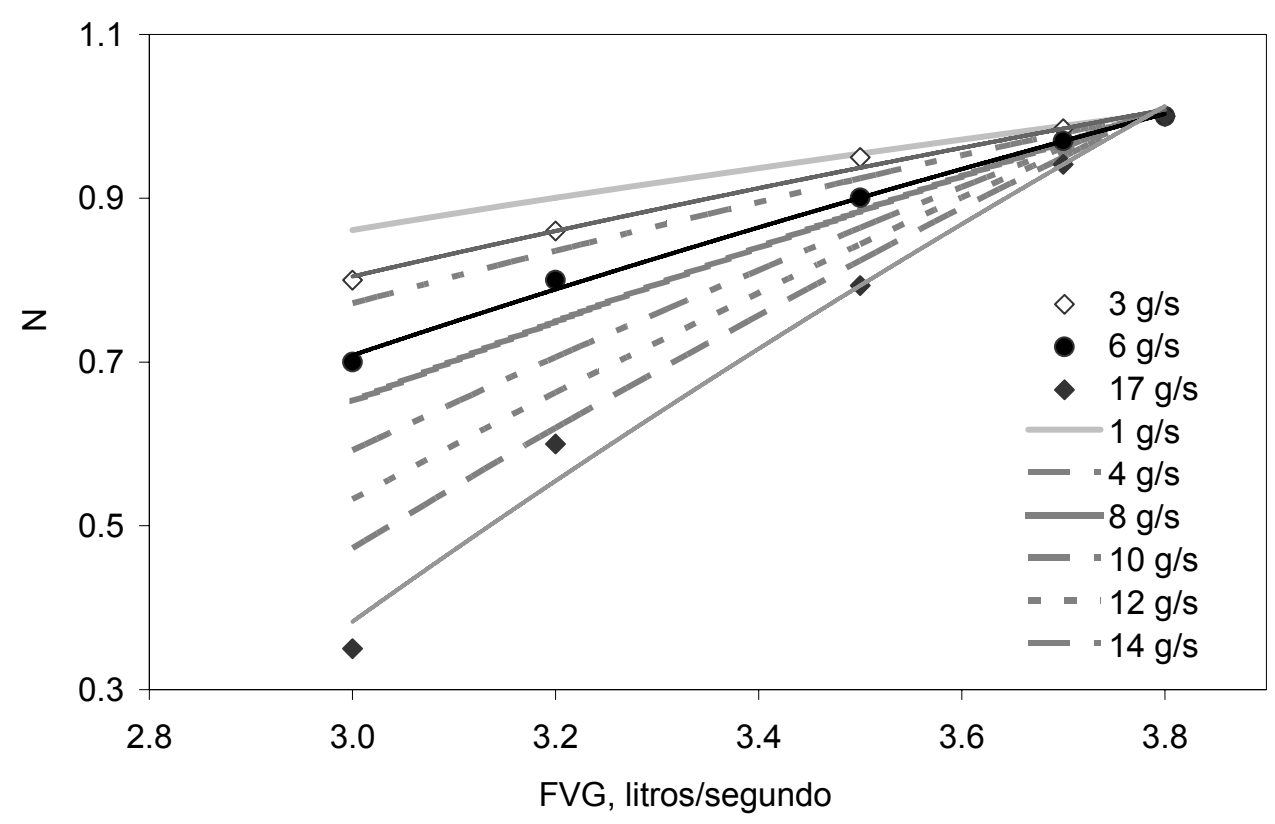

Fig. 8: Agitación que se obtiene en una etapa, como función del flujo volumétrico del gas, para diferente flujo másico de sólidos.

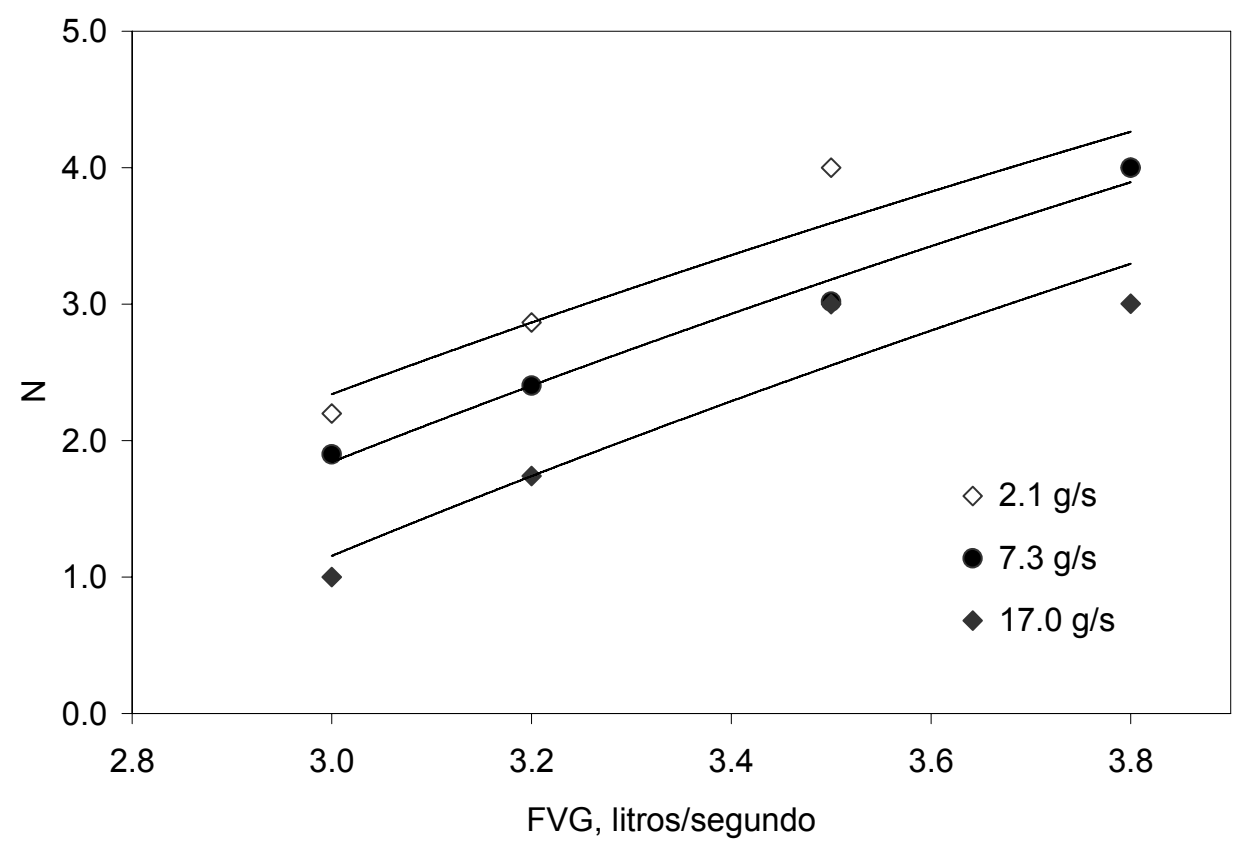

Fig. 9: Número de tanques en serie, $\mathrm{N}$, como función de los flujos de gas y sólidos para la columna de tres etapas. $\diamond 2.1 \mathrm{~g} / \mathrm{s}, \mathrm{y}=8.1358 \ln (\mathrm{x})-6.5972$, $R^{2}=0.8932, \bullet 7.3 \mathrm{~g} / \mathrm{s}, \mathrm{y}=8.6745 \ln (\mathrm{x})-7.6865, \mathrm{R}^{2}=0.9833 ; \bullet 17 \mathrm{~g} / \mathrm{s} \mathrm{y}=$ $9.0544 \ln (x)-8.7913, R^{2}=0.8942$. 


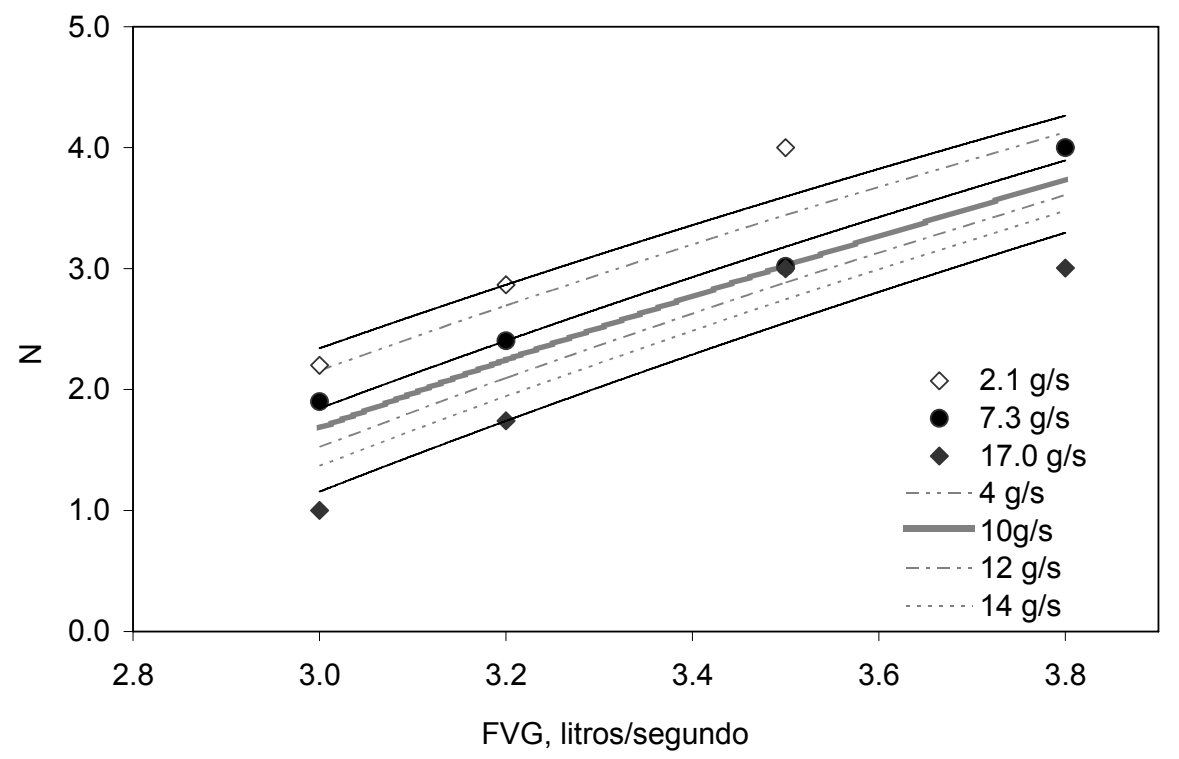

Fig. 10: Influencia del flujo volumétrico del gas y del flujo másico de sólidos en la agitación (número de tanques agitados, $\mathrm{N}$ para un sistema de tres lechos fuente.

\section{CONCLUSIONES}

Los cuatro modelos RTD considerados y aplicados en este trabajo, muestran bajo que condiciones de operación se puede obtener un tratamiento más uniforme de las partículas sólidas; estas condiciones de operación son como era de esperarse- aquellas en las que se utiliza el mayor flujo de gas y el menor flujo de sólidos.

Los experimentos con la columna de tres etapas fueron de gran utilidad para demostrar, utilizando el modelo de tanques-en-serie, que los lechos fuente en serie permiten un tratamiento uniforme de los sólidos, acercándose esta corriente al flujo pistón a medida que el número de etapas aumenta.

En el análisis estadístico de los modelos de compartimentos aquí presentados, se demuestra que no existe diferencia estadísticamente significativa en el ajuste de los datos experimentales respectivos, indicando con ello, que el más recomendable de los modelos debido a su simplicidad, es el modelo de compartimentos propuesto por Arriola (1997).

\section{NOMENCLATURA}

$\mathrm{d}_{\mathrm{p}}=$ diámetro característica de la partícula, ( $\mathrm{mm}$ )

$\mathrm{N}=$ Número de tanques agitados (modelo de tanques en serie)

\footnotetext{
$\mathrm{t} \quad=$ Tiempo (s)

$\overline{\mathrm{t}}=$ Tiempo promedio de residencia, (s). ecns. (27).

$\mathrm{C}=$ Concentración del rastreador, $(\mathrm{g}$ rastreador en la muestra/ $\mathrm{M}$ )

$\mathrm{E}(\mathrm{t})=$ Función de distribución de tiempos de residencia RTD, $\left(\mathrm{s}^{-1}\right)$

$\mathrm{E}(\mathrm{t})_{1}=$ Función de distribución de tiempos de residencia RTD para una etapa, $\left(s^{-1}\right)$

$\mathrm{E}(\mathrm{t})_{3}=$ Función de distribución de tiempos de residencia RTD para tres etapas, $\left(\mathrm{s}^{-1}\right)$

$\mathrm{E}(\mathrm{t})_{\mathrm{n}} \quad=$ Función de distribución de tiempos de residencia $R T D$ para $n$ etapas, $\left(s^{-1}\right)$

FVG = Flujo volumétrico del gas, (L/s)

$\mathrm{M}=$ Masa del rastreador total, $(\mathrm{g})$

$\mathrm{N}=$ Número de etapas

$\mathrm{S}_{\mathrm{o}} \quad=$ Flujo másico de los sólidos, (g/s),

$\mathrm{W}=$ Peso total del lecho, $(\mathrm{g})$

CSTV = Siglas en inglés de los recipientes continuos de tanque agitado

$\mathrm{D} / \mathrm{uL}=$ Módulo de dispersión

$\mathrm{A}=$ Constante

$\mathrm{B}=$ Constante

a-f $\quad=$ Coeficientes de la fracción parcial

$r \quad=\quad$ Fracción de volumen del primer CSTV
} 


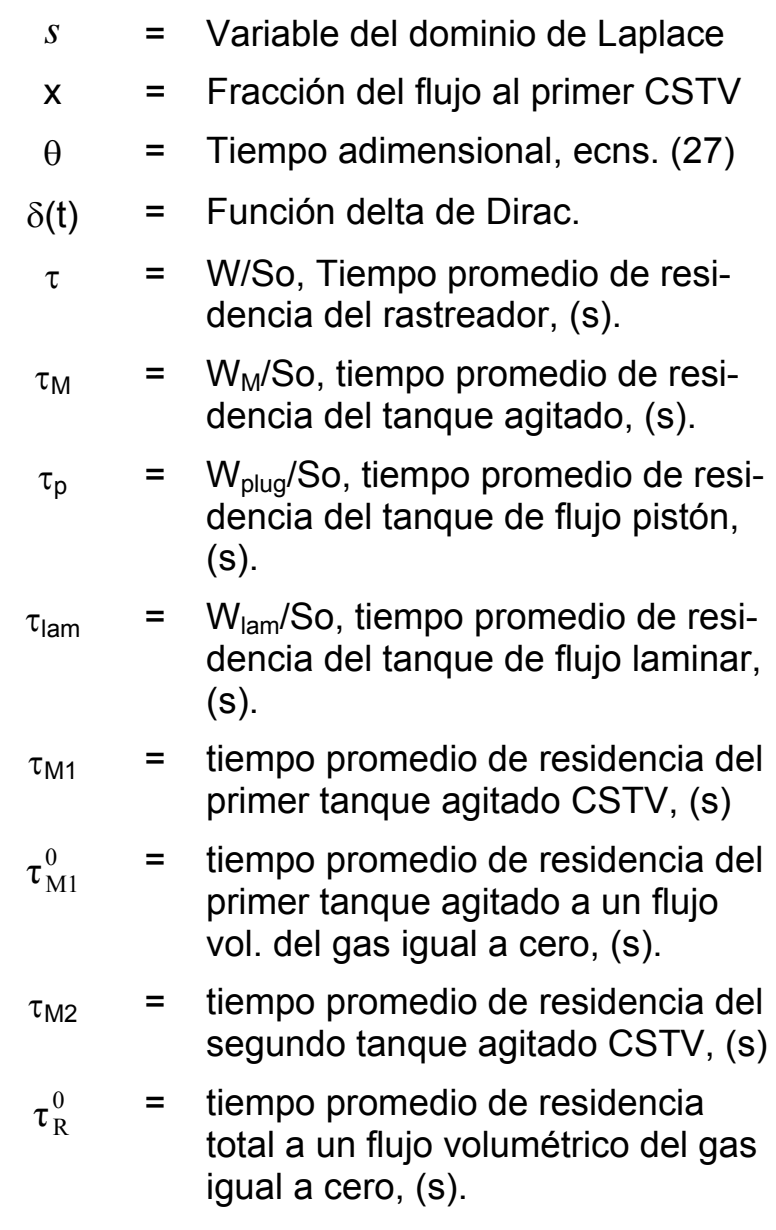

\section{AGRADECIMIENTOS}

Los autores agradecen al Centro de Investigación y Asistencia en Tecnología y Diseño del Estado de Jalisco, A. C., a la Universidad de Guadalajara, y al Consejo Nacional de Ciencia y Tecnología, beca No.138386, México.

\section{REFERENCIAS}

Aguado, R., M. Olazar, B. Gaisán, R. Prieto y J. Bilbao, Kinetics of polystyrene pyrolysis in a conical spouted bed reactor, Chemical Engineering Journal, 92(1), 91-99, (2003).

Arandes, J. M., J. Bilbao, D. López-Valerio, Reciclado de Residuos Plásticos, Revista Iberoamericana de Polímeros, 5(1), 10-11, (2004).

Arriola, E., C. F. Cruz-Fierro, K. H. Alkhaldi, B. P. Reed y G. N. Jovanovic, Residence Time Distributions in Staged Spouted Beds, Canadian J. of Chem. Eng., 82(2), 94-101, (2004).

Arriola, E., Residence Time Distribution of Solids in Staged Spouted Beds, Ph.D. thesis,
Oregon State University, Corvallis, Oregon, USA, (1997).

Banks, H. J., Prospects for heat disinfestations, Stored Grain Research Laboratory, CSIRO Entomology, GPO Box 1700, Canberra, ACT 2601, http://www.sgrl.csiro.au/aptc1998/50_banks.p df, (1998).

Bacelos, M. S., P.I. Spitzner, A.M. Silveira y J.T. Freire, Analysis Of Drying Of Pastes In Spouted Beds, Proceedings of the 14th International Drying Symposium, São Paulo, Brazil, C, 1537-1544, (2004).

Beckett, S. J. y E. J., Wright, Increasing Heating Rate $Y$ Decreasing Disinfestation Temperature For More Affordable Heat Disinfestation Of Grain, Stored Grain Research Laboratory, CSIRO Entomology, G.P.O. Box 1700, Canberra, A.C.T 2601, Australia, (2000).

Benali, M. y M., Amazouz, Drying of Liquid Biomaterials in a Jet Spouted Bed of Teflon Particles, 52nd Canadian Chemical Engineering Conferences, Vancouver, Canada, October (2002).

Clary, B. L., K. K. Agrawal, y G. L., Nelson, Simultaneous Heat And Mass Transfer From Peanuts In A Spouted Bed, Meeting Amer. Soc. of Agr. Eng., Chicago, Paper No. 70-308. ASAE, St. Joseph, Michigan, USA, (1970).

Chan L. W., E. S. K. Tang y W. S. P. Heng, Comparative Study of the Fluid Dynamics of Bottom Spray Fluid Bed Coaters, AAPS Pharm. Sci. Tech., 7(2), Article 372006, E1E9, (2006).

Elperin, I. T. y B. K. Khokhlov, Nauch, Soobshchem, NITsEMENT, 20, 51, (1965).

Epstein, N. y K. B. Mathur, Spouted Beds, Academic Press: New York, U.S.A, (1974).

Epstein, N. y K. B. Mathur, Applications of Spouted Beds, Section 8.5.6 en "Handbook of Multiphase Systems" (Ed. G. Hetsoni), Hemisphere, (1982).

Guatemala, G. M., Modelos de Flujo No-Ideal para Lechos Fuente, Universidad de Guadalajara, Tesis de Maestría, México, (2001).

Información Tecnológica - Vol. 18 Nº 1 - 2007 
Grbavcic, Z.B., D.V. Vukovic, F. K. Zdanski, H. Littman, Fluid Flow Pattern, Minimum Spouting Velocity And Pressure Drop In Spouted Beds, Can. J. Chem. Eng., 54, 3342, (1976).

Jones, D., Air suspensión coating for multiparticulates, Drug Development And Industrial Pharmacy, 20(20), 3175-3206, (1994).

Krzywanski, R.S., N. Epstein y B. D. Bowen, Multi-Dimensional Model of a Spoouted bed, Can. J. Chem. Eng., 70, 858-872, (1992).

Levenspiel, O., Ingeniería de las Reacciones Químicas, 3a Ed., 448-451, Limusa Wiley, México, (2004).

Levenspiel, O., The Chemical Reactor Omnibook, Distributed by: OSU Book Stores, Inc. Corvallis Oregon 97339, 66.1-66.2, January, USA, (1993).

Levenspiel, O. y K. B. Bishoff, Patterns of Flow In Chemical Process Vessels, Advan. Chem. Eng., 4, 95, USA, (1963).

Lim, C.J. y K, B. Mathur, A Flow Model For Gas Movement In Spouted Beds, AIChE J., 22, 674-680, (1976).

Littman, H., M.H. Morgan, V.N. Pallassana, S. J. Kim, J.Y. Day y G.M. Lazarek, An Axisymmetric Model Of Flow In The Annulus Of A Spouted Bed Of Coarse Particle. Model Experimental Verification And Residence Time Distribution, Can. J. Chem. Eng., 63, 188-194, (1985).

Malek, M.A. y J.H. Walsh, The Treatment of Coal For Coking By The Spouted Bed Process, Department of Mines and Technical Surveys Mines Branch, Ottawa, Division Report FMP 66/54-SP, (1966).

Mann, U. y E. J., Crosby, Modeling Circulation Of Solids In Spouted Beds, Ind. Eng. Chem. Process Des. Develop., 11(2), 314-316, (1972).

Murthy, D.V.R. y P.N. Singh, Minimum Spouting Velocity In Multiple Spouted Beds, Can. J. Chem. Eng., 72, 235-239, (1994).

Nagahashi, Y., J.R. Grace, D.H. Lee y Y. Asako, Enhancement Of Spouting And Solids
Circulation Of A Gas_Spouted Bed By Addition of Small Quantities of Liquid, 52nd Canadian Chemical Engineering Conferences, October, Vancouver, Canada, (2002).

Nagaraju, V. D., C. T., Murthy, K., Ramalakshmi y P. N., Srinivasa Raoa, Studies on Roasting of Coffee Beans in a Spouted Bed, Journal of Food Engineering, 31, (2), 263-270, (1997).

Olazar, M., M. J. San José, F. J. Peñas, A. T. Aguayo, J. M. Arandes y J. Bilbao, A Model for Gas Flow in Jet Spouted Beds, Can. J. Chem. Eng., 71, pp 189-194, (1993).

Olazar, M., M. J. San Jose, G Zabala y J. Bilbao, A New Reactor in Jet Spouted Bed Regime for Catalitic Polimerizations, Chem Eng. Sci., 49, 4579-4588, (1994).

Olazar, M, M. J. San Jose, S. Alvarez, A. Morales y J. Bilbao, Measurement of Particle Velocities in Conical Spouted Beds Using an Optical Fiber Probe, Ind. Eng. Chem. Res., 37, 4520-4527, (1998).

Peterson, W. S., Spouted Bed Drier, Can. J. Chem. Eng., 40, 226, (1962).

Rovero, G. y A. P. Watkinson, A Two-Stage Spouted Bed Process For Autothermal Pyrolysis Or Retorting, Fuel Processing Technology, 26, 221, (1990).

San José, M. J., M. Olazar, S. Alvarez, A. Morales y J. Bilbao, Local Porosity In Conical Spouted Beds Consisting Of Solids Of Varying Density, Chemical Engineering Science, 60, 7, 2017-2025, (2005).

Souza, C. R. F. y W. P. Oliveira, Spouted Bed Drying Of Bauhinia Forficata Link Extract: The Effects Of Feed Atomizer Position And Operating Conditions On Equipment Performance And Product Properties, Braz. J. Chem. Eng., 22, 2, 239-247, (2005).

Tapaneyasin, R., S. Devahastin y A. Tansakul, Drying Methods And Quality Of Shrimp Dried In A Jet-Spouted Bed Dryer, Journal of Food Process Engineering, Blackwell Publishing, 28, 35-52, (2005).

Tsvik, M. Z., M. N., Nabiev, N. U., Rizaev, K. V., Merenkov, y V. S., Vyzgo, The velocity for external spouting in the combined process for 
production of granulated fertilizers, Uzb. Khim. Zh., 11(2), 50, (1967).

Uemaki, O. y M. Kugo, Heat Transfer In Spouted Beds, Kagaku Kogaku, 31, 348, (1967).

Van Weert, G. y J. B. Van Hasselt, Countercurrent Flow of Dissimilar Solids in a Vertical Multistage, Spouting Bed Configu- ration, paper presented at The Richard Mozley Memorial Symposium, Falmouth, Cornwall, U. K., June 4-5, (1997).

Wang, Z. G., H. T. Bi y C. J. Lim, Minimum Spouting Velocities in Conical Spouted Beds, 52nd Canadian Chemical Engineering Conferences, October, Vancouver, Canada, (2002). 